\title{
The influence of resistance and carrier concentration on the output voltage of a $\mathrm{ZnO}$ nanogenerator
}

\author{
Thomas S van den Heever ${ }^{*}$ and Willem J Perold
}

\begin{abstract}
Zinc oxide ( $\mathrm{ZnO}$ ) nanowires were synthesised through two different methods; the vapour liquid solid (VLS) method and an aqueous solution method. Each method had its own set of parameters and changes in those parameters influenced the output voltage of a nanogenerator. Changes in output voltage occurred as a result of variations in the resistance and the carrier concentration of grown nanowires as the piezoelectric charges were screened or trapped. An optimal value for both the resistance and the carrier concentration was determined in order to optimise the output voltage.
\end{abstract}

Keywords: Output voltage; Nanogenerator; ZnO nanowires; Carrier concentration

\section{Introduction}

Research into zinc oxide $(\mathrm{ZnO})$ thin films and nanowires have been increasing over the last couple of years. The research has led to the development of numerous $\mathrm{ZnO}$ thin film and nanowire based devices ranging from surface acoustic wave filters [1], photonic crystals [2], light emitting diodes [3], photodetectros [4], photodiodes [5], gas sensors [6] and solar cells [7], to name a few. This is due to the unique properties of $\mathrm{ZnO}$ including a wide bandgap $(3.37 \mathrm{eV})$ as well as specific electrical and optoelectrical properties of the II-VI semiconductor group [2-4].

A new application of $\mathrm{ZnO}$ nanowires is the generation of electricity by converting an applied mechanical force to electricity through the piezoelectric effect [8]. In recent years various other materials were also used to manufacture nanogenerators, these materials include $\mathrm{BaTiO}_{3}$ $[9,10]$. In the process of manufacturing the nanogenerators, the nanowires are synthesised by various methods and on various substrates.

For the purpose of this work the $\mathrm{ZnO}$ nanowires are synthesised using two methods: The vapour liquid solid (VLS) method and an aqueous solution method. The nanowires are grown only on n-type silicon (100) substrates in order to compare the two growth methods. Both

*Correspondence: tsvdh@sun.ac.za

Stellenbosch Univeristy, Stellenbosch, Western Cape, 7600, South Africa methods are widely used and the exact details on the growth mechanism can be found elsewhere [11-13].

\section{Methodology}

In short, the VLS method consists of a horizontal tube furnace operating at high temperature, typically in the range of $1000^{\circ} \mathrm{C}$. The source material, a $1: 1$ weight ratio of $\mathrm{ZnO}$ and graphite powders, are loaded in the centre of a quartz tube that is then loaded into the furnace, with the substrate, where the growth will take place, a fixed distance from the source powders. The substrate is coated in a seed-layer that acts as a catalyst during the growth. The quartz tube is placed under vacuum and the furnace is heated up to a fixed temperature, around $1000^{\circ} \mathrm{C}$. A carrier gas, argon, is introduced to the system to carry the evaporated source materials to the substrate, when the furnace reached the desired temperature [11,12].

In contrast to the VLS method, the aqueous solution method works at much lower operating temperature, below $100^{\circ} \mathrm{C}$. Zinc nitrate hexahydrate, a zinc salt, and hexamethylenetetramine (HMTA) is dissolved in a 1 : 1 equimolar solution in DI-water. The synthesis is conducted by the aqueous thermal decomposition of $\mathrm{Zn}^{2+}$ amino complex with reagent-grade chemicals. The chemicals react within the solution to form $\mathrm{ZnO}$ nanowires on the pretreated substrates that are floating on top of the solution $[13,14]$.

\section{Springer}

(C) 2013 van den Heever and Perold; licensee Springer. This is an Open Access article distributed under the terms of the Creative Commons Attribution License (http://creativecommons.org/licenses/by/2.0), which permits unrestricted use, distribution, and reproduction in any medium, provided the original work is properly cited. 
The growth for both methods is carried out on n-type $\mathrm{Si}$ (100) substrates that are cleaned and a thin layer of $\mathrm{ZnO}(20 \mathrm{~nm})$ is deposited via RF-magnetron sputtering and acts as a seed-layer for the nanowire growth. The nanowire growth is characterised using a scanning electron microscope (SEM). The Phenom Fei SEM [15] was used to examine the nanowires at different magnification, after growth. The growth direction, growth density and nanowire morphology was examined and compared between the two different nanowire growth methods.

The output voltage of the $\mathrm{ZnO}$ nanowire substrate is measured by placing a gold electrode on top of the nanowires, and a force is then applied to this electrode. The applied force results in the electrode bending the nanowires, which in turn creates a piezoelectric potential and as a result of the formation of a Schottky contact at the nanowire electrode interface, an output voltage is observed [16]. The applied force can be controlled by placing various weights on the electrode to ensure accurate measurements are taken. The resistance and the carrier concentration of the nanowire arrays are measured, using the Van der Pauw and Hall methods respectively $[17,18]$.

During the growth of the $\mathrm{ZnO}$ nanowires certain parameters were changed in consecutive runs to see the influence each parameter has on the growth. Table 1 shows the parameters, with the maximum and the minimum levels of the parameters that are changed during the VLS growth. Table 2 shows a similar table for the aqueous solution method.

During the growth of the nanowires the parameters were changed, one at a time, between the specified levels in Table 1 and Table 2. The influence that the change in growth parameters has on the nanowire growth is observed with the SEM and the carrier concentrations, the resistance and the output voltage values are measured. High magnification images are used to observe the difference in nanowire morphology and lower magnification images are used to observe the growth density and growth direction.

The substrate with grown nanowires is fixed to a testboard shown in Figure 1. The substrate, with nanowires grown on top is placed in the centre and the corners are

Table 1 Different growth parameters, with high and low levels, used during the VLS growth of $\mathrm{ZnO}$ nanowires

\begin{tabular}{lcc}
\hline \multicolumn{1}{c}{ Parameter } & Low level & High level \\
\hline Growth time (minutes) & 5 & 30 \\
\hline Growth temperature $\left({ }^{\circ} \mathrm{C}\right)$ & 500 & 1200 \\
\hline Initial pressure (mTorr) & 200 & 2000 \\
\hline Growth pressure (mTorr) & 500 & 1500 \\
\hline Source powder (grams) & 1 & 3 \\
\hline Argon flow rate (sccm) & 20 & 120 \\
\hline
\end{tabular}

Table 2 Different growth parameters, with high and low levels, used during the aqeuous solution growth of $\mathrm{ZnO}$ nanowires

\begin{tabular}{ccc}
\hline Parameter & Low level & High level \\
\hline Growth time (hours) & 1 & 24 \\
\hline Growth temperature $\left({ }^{\circ} \mathrm{C}\right)$ & 60 & 120 \\
\hline Concentration $(\mathrm{mM})$ & 10 & 100 \\
\hline Covered & No & Yes \\
\hline Ratio (Zinc salt : HMTA) & $1: 0.75$ & $1: 1.25$ \\
\hline
\end{tabular}

connected to the conducting lines using silver paste. The silver paste is put on top of the nanowires connecting the nanowires to the test-board. Conducting wires are added to the silver paste and is connected to the measuring equipment.

The Van der Pauw method is used to measure the resistivity of the nanowire substrate. The substrate is put in place with each corner numbered as shown in Figure 1. In total eight different resistance values are calculated by applying current through two terminals and measuring the voltage across the remaining two terminals. Resistances $R_{12,34}, R_{34,12}, R_{21,43}, R_{43,21}, R_{23,41}, R_{41,23}, R_{32,14}$ and $R_{14,32}$ are measured and is generically defined as $R_{a b, c d}=$ $V_{c d} / I_{a b}$, with $a, b, c$ and $d$ integers between 1 and 4 . The average of the first four values is defined as $R_{v}$, while the average of last four values gives $R_{h}$. The resulting resistance, $R_{s}$, of the nanowire substrate is derived from the Van der Pauw equation [17]

$$
e^{-\pi R_{v} / R_{s}}+e^{-\pi R_{h} / R_{s}}=1
$$

The carrier concentration is measured by placing the nanowire substrate in a constant magnetic field, and by application of a constant current, the Hall voltage, $V_{h}$, is measured. The substrate, fixed to the test-board, is placed

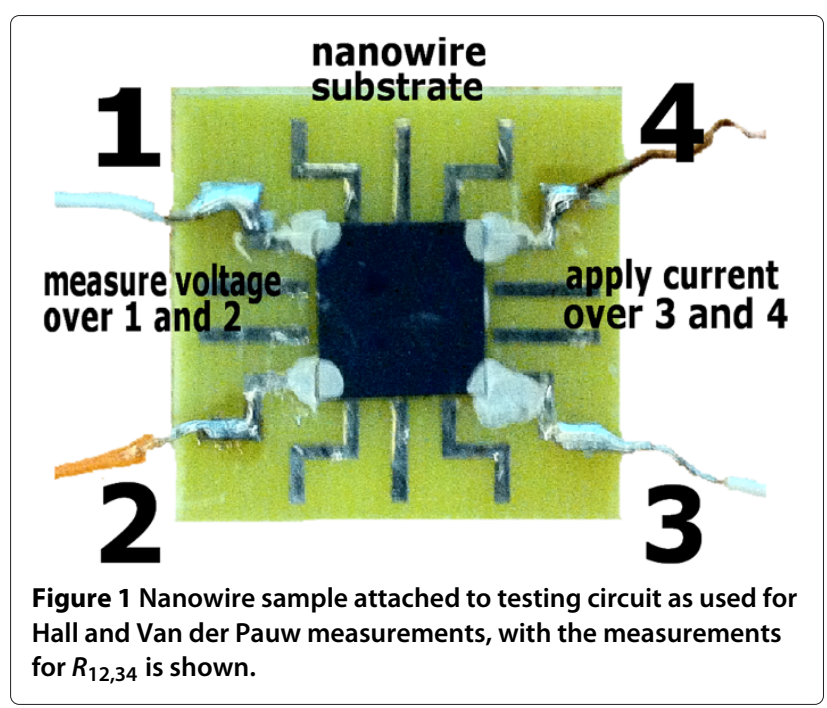


in the magnetic field and a constant current is applied diagonally. The currents $I_{13}, I_{31}, I_{24}$ and $I_{42}$ are applied consecutively and the resulting voltages are measured over the open terminals $V_{42}, V_{24}, V_{13}$ and $V_{31}$, respectively. The magnetic field is then reversed and the same measurements are again made. The voltages measured in the negative oriented magnetic field are subtracted from the voltages in the positive oriented magnetic field. The carrier concentration is calculated using a modified version of the Hall equation [16]

$$
n_{s}=\frac{8 \times 10^{-8} I B}{q d\left[V_{24}+V_{42}+V_{13}+V_{31}\right]}
$$

where $I$ is the constant current in Ampere, $B$ is the magnetic field strength in Gauss, $q$ is the electron charge and $d$ is the depth of the material.

Normally the Hall and Van der Pauw methods are used for thin films, but the nanowire growth is so dense that it can be modelled as a thin layer. Ideally impedance spectroscopy should be used to measure the resistance and carrier concentration of the nanowire arrays. Although the exact values generated from these two methods might not be as accurate, the findings are in line with theoretical predeictions. The Hall method has previously been used to measure these quantities with great success [19]. More accurate measurements are required to further confirm the results presented in this article.

The constant current is applied using the $120 \mathrm{~B}$ Constant Current source form Lake Shore, Ohio, USA. Voltages are measured using a digital voltmeter.

The resistance measurements were made with a constant current flow of $10 \mu \mathrm{A}$. The Lakeshore current source has a compliance voltage of $11 \mathrm{~V}$ and in order to ensure this voltage is never exceeded, all test were conducted at a low current. The resistance values of the samples ranged from a few $100 \mathrm{ohm}$ which corresponds to no nanowire growth, to just below $1 \mathrm{M} \Omega$ which corresponds to very dense nanowire growth.

The carrier concentrations are measured at a $10 \mu \mathrm{A}$ constant current, with a constant magnetic field of 3700 Gauss. Measurements were made with the magnetic field applied along the positive $\mathrm{z}$-axis. The field was then reversed and the measurements repeated. The carrier concentrations are expected to range from $10^{14}$ to $10^{20}$, as predicted by Wang et al. [20,21].

\section{Findings}

Figure 2(a) shows typical nanowire growth using the VLS method. The nanowires are $60-100 \mathrm{~nm}$ in diameter and 3-4 micrometres in length. Figure 2(b) shows typical growth achieved using the aqueous solution method. The nanowires are 100-150 $\mathrm{nm}$ in diameter and 1.5-2 micromeres in length.
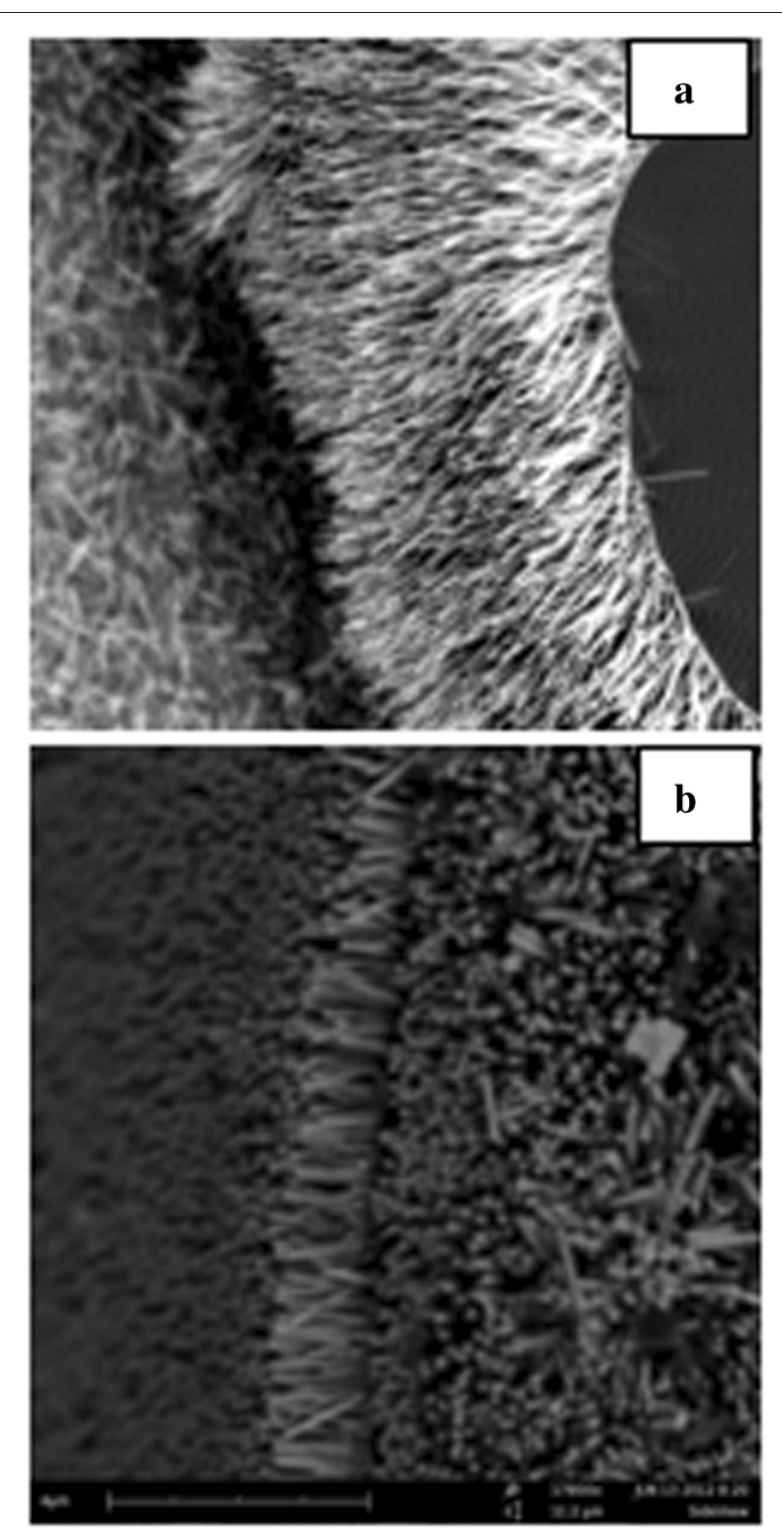

Figure 2 Typical nanowire growth by means of the a) VLS method, and b) the aqueous solution growth method.

The VLS nanowire growth results in longer and thinner nanowires, but the growth direction is more random compared to the aqueous solution method. The nanowires are not aligned as well as in the aqueous solution growth. The aqueous solution grown nanowires are aligned perpendicular to the substrate. The growth also appears to be more dense than that of the VLS growth.

Over 120 samples were grown, more than 30 with the VLS method and 90 using the aqueous solution method. Each sample was examined with the SEM at different magnification to look at the nanowire morphology, growth direction and growth density. Overall the growth of the two methods looked similar, however, at times the VLS 
method resulted in nanowire growth with random growth directions. Each sample was placed on a test board in order to measure the resistance and carrier concentration. All the data is obtained using both growth methods.

Figure 3 shows the relationship between the measured resistance and carrier concentration values. From the graph it is clear that at low resistance the carrier concentration is high. This is expected because at low resistance there are a lot of charge carriers for current transport. At higher resistance the number of carriers decreases which means the current cannot propagate through the material as easy and hence the resistance is higher. Some points do not fall on the fitted graph and this is due to two main factors. Firstly all the samples were not grown on the same silicon wafer, but rather a couple of different wafers were used to prepare all the samples. The intrinsic carrier concentration of the silicon wafers can be different and this leads to slightly higher measured carrier concentrations. Secondly, the deposition of the $\mathrm{ZnO}$ layer cannot be controlled within nanometer accuracy and hence the thickness could vary. A couple of extra nanometers of $\mathrm{ZnO}$ would also increase the carrier concentration.

The biggest problem with using the Hall method to measure the carrier concentration of nanowires is that the method was developed for thin films. In this films the current can flow freely in thin two dimensions of the film. With the nanowires a third demension is added and the current might not travel through the nanowires but it might travel through the base of the nanowires leading to inaccurate measurements. Although this might be the case, the resulting values are in the theoretically predicted range and hence the method was used for further investigation.

As mentioned, due to the piezoelectric effect, when the $\mathrm{ZnO}$ nanowires are bent, a piezoelectric potential is created. With the presence of a Schottky contact the piezoelectric potential can be measured as the output voltage of a nanogenerator [16].

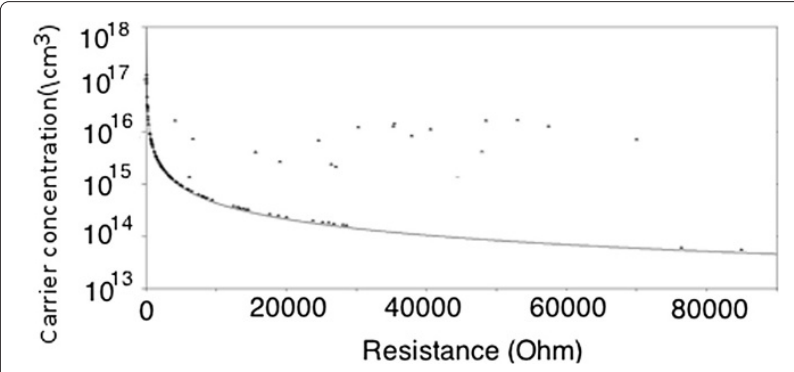

Figure 3 Relationship between the resistance and carrier concentration of grown $\mathrm{ZnO}$ nanowire samples. The fitted graph shows at low resistance the carrier concentration is high and at high resistance the carrier concentration is low, as obtained from both VLS and aqueous solution growth.

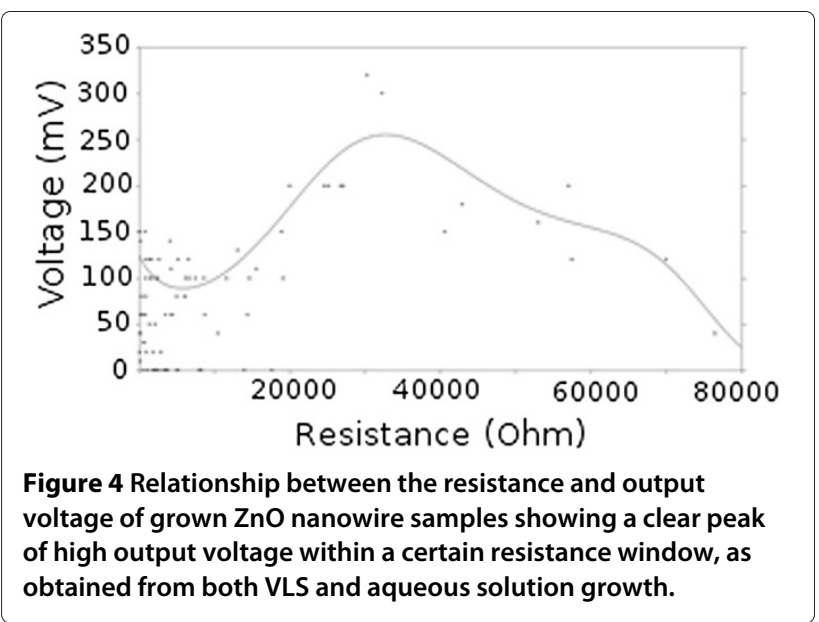

According to Wang et al. the generated piezoelectric potential can be screened by the charge carriers $[20,21]$. This will lead to a reduction in size of the output voltage, but it will not fully screen the piezoelectric potential. Under ideal conditions the charge carriers will be absent, and thus the piezoelectric potential is not screened at all, resulting in a maximum output voltage. The problem however is with no charge carriers the piezoelectric charge will be trapped inside the material due to the high resistance. If, in this ideal case however, the carrier concentration is too large, the charges will screen the piezoelectric charge, the higher the carrier concentration the more the piezoelectric charge is screened resulting in a much lower voltage $[20,21]$. Somewhere is between these two extreme cases an optimal value for carrier concentration will exist. The concentration will be high enough to allow good conductivity and low resistance but still be low enough as to not screen the piezoelectric charge too much.

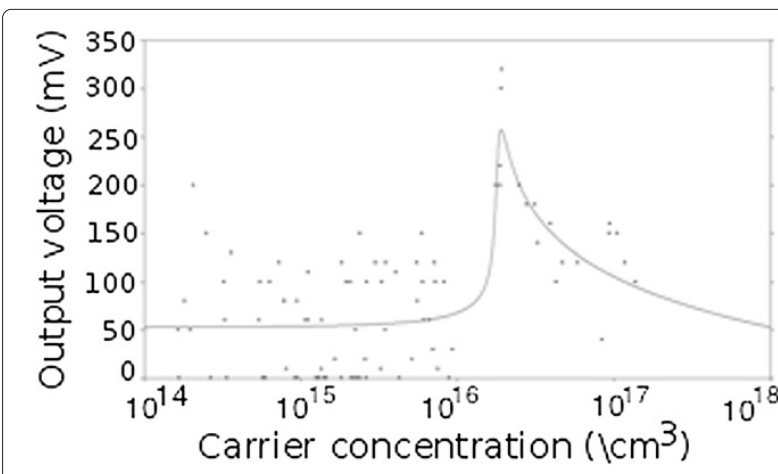

Figure 5 Relationship between the carrier concentration and output voltage of grown $\mathrm{ZnO}$ nanowire samples, showing a clear peak in output voltage at a specific carrier concentration, as obtained from both VLS and aqueous solution growth. 
Figure 4 shows the relationship between the resistance and the output voltage of the grown $\mathrm{ZnO}$ nanowire samples. At low resistance the output voltage is low due to the high carrier concentration, which leads to a large screening of the piezoelectric potential. At high resistance the output voltage is low due to the internal losses that will occur. A larger voltage drop is observed internally, over the nanowires, which results in a lower output voltage. A clear peak is visible where a balance exists between high carrier concentration and internal losses. As predicted by Wang et al., an optimal range exists where the carrier concentration and resistance are at values that lead to optimal output voltage.

There are more samples with a lower resistance compared to the higher end. The VLS method yielded high resistance samples and there were less VLS samples compared to the aqeuous solution samples [22,23]. Hence, there are less data points in the higher resistance part of the graph.

The final important relationship is shown in Figure 5, where the carrier concentration and output voltage graph is shown. As explained above, at low carrier concentration the piezoelectric charges are not free to move and a lot of losses occur internally. At high carrier concentration the piezoelectric charges are screened which results in a lower output voltage. Somewhere in between these two extreme cases an optimal value for carrier concentration exists, as seen in Figure 5. Figure 4 and Figure 5 shows exactly what Wang et al predicted: an optimal value of carrier concentration exists where the piezoelectric charges are screened and the resistance is still low enough to endure high output voltage [20,21].

The morphology and size of the nanowires influences the functionality and parameters of the nanowire, including the output voltage $[24,25]$. The average output voltage of the nanowires grown via the aqueous solution method is just above $150 \mathrm{mV}$, compared to just above $80 \mathrm{mV}$ for the VLS grown nanowires.

The main reason for the difference in the measured output voltage is argued to be the difference in the growth direction of the nanowires. The VLS method yielded nanowires that were not aligned but rather grew in random directions, compared to the aqueous solution method which yields vertically aligned nanowires. When the nanowires are bend by the electrode the piezoelectric charges are polarized in the longitudinal direction of the nanowire [26]. This means that the piezoelectric charge in vertically aligned nanowires will be higher when compared to unaligned nanowires. This is the case where the aqueous solution growth yield higher output voltages when compared to the VLS method.

As mentioned, the Hall measurements were designed for solid thin films. The above results are for nanowires that are approximated as thin films as an easy method to measure the carrier concentration does not exist. The exact values of the carrier concentrations might differ from the true values but the overall trend will be the same. The results coincides with the theoretical findings of Wang et al. [20,21].

\section{Summary}

$\mathrm{ZnO}$ nanowires were prepared via two different methods, VLS and an aqueous solution method. The carrier concentration of the grown nanowires greatly influences the resistance of the nanowires as well as the output voltage. An optimal value for the carrier concentration exists, where it is high enough to ensure good conductivity, but low enough as to not screen the piezoelectric charge. On average the output voltage of the nanowires grown with the aqueous solution method is higher than the nanowires grown by the VLS method, due to the aligned growth of the aqueous grown nanowires. The exact values measured here might also differ from the true results because Hall measurements were designed for solid thin films and the nanowires only approximate thin films.

\section{Competing interests}

Both authors declare that they have no competing interests.

\section{Authors' contributions}

TS van den Heever conducted the research presented in this article from the growing of the nanowires to the measurement of all the parameters presented. WJ Perold gave the necessary technical background and guidance for the work presented. Both authors read and approved the final manuscript.

Received: 20 May 2013 Accepted: 23 August 2013

Published: 11 September 2013

\section{References}

1. Emanetoglu NW, Gorla C, Liu Y, Liang S, Lu Y (1999) Epitaxial ZnO piezoelectric thin films for SAW filters. Mater Sci Semicond Process 2: 147

2. Chen Y, Bagnall D, Yao T (2000) ZnO as a novel Photonic material for the UV Region. Mater Sci Eng B 75: 190

3. Saito N, Haneda H, Sekiguchi T, Ohashi N, Sakaguchi I, Koumoto K (2002) Low-temperature fabrication of light-emitting zinc oxide micropatterns using self-assembled monolayers. Adv Mater 14: 418

4. Laing S, Sheng H, Liu Y, Hio Z, Lu Y, Shen H (2001) ZnO Schottky ultraviolet photodetectors. J Cryst Growth 225: 110

5. Lee JY, Choi YS, Kim JH, Park MO, Im S (2002) Optimizing n-ZnO/p-Si heterojunctions for photodiode applications. Thin Solid Films 403: 533

6. Golego N, Studenikin SA, Cocivera M (2000) Sensor photoresponse of thin-film oxides of zinc and titanium to oxygen gas. J Electrochem Soc 147: 1592

7. Keis K, Magnussin E, Lindstrom H, Linquist SE, Hagfeldt A (2002) A 5\% efficient photo electrochemical solar cell based on nanostructured $\mathrm{ZnO}$ electrodes. Sol Energy 73: 51

8. Hu Y, Zhang Y, Xu C, Lin L, Snyder RL, Wang ZL (2011) Self-powered system with wireless data transmission. Nano Lett 11: 2572

9. Park K, Xu S, Liu Y, Hwang G, Kang SL, Wang ZL, Lee KJ (2010) Piezoelectric BaTiO3 thin film nanogenerator on plastic substrates. Nano Lett 10: 4939

10. Park K, Lee M, Liu Y, Moon S, Hwang G, Zhu G, Kim JE, Kim SO, Kim DK, Wang ZL, Lee KJ (2012) Flexible nanocomposite generator made of BaTiO3 nanoparticles and graphitic carbons. Adv Mater 24: 2999

11. Huang MH, Wu Y, Feick H, Tran N, Weber E, Yangm P (2001) Catalytic growth of zinc oxide nanowires by vapor transport. Adv Mater 13: 113

12. Pan ZW, Dai ZR, Wang ZL (2001) nanobelts of semiconducting oxides. Science 291: 1947 
13. Vayssieres $L$ (2003) Growth of arrayed nanorods and nanowires of $\mathrm{ZnO}$ from aqueous solutions. Adv Mater 15: 464

14. Vayssieres L, Keis K, Lindquist S, Hagfeldt A (2001) Purpose built anisotropic metal oxide material: 3D highly oriented microrod array of ZnO. J Phys Chem B 105: 3350

15. Desktop SEM solutions. Information on http://www.phenom-world.com

16. van den Heever TS, Perold WJ, Buttner U (2011) A novel method to measure the generated voltage of a $\mathrm{ZnO}$ nanogenerator. Nanotechnol 22: 395204

17. van der Pauw $L J$ (1958) A method of measuring the resistivity and hall coefficient on lamellae of Arbitary shape. Philips Tech Rev 59: 220

18. Hall EH (1879) On a new action of the magnet on electric currents. Am J Math 2: 287

19. Meyer D (2008) Phd thesis, Surface Passivation studies of AlGaN/GaN high electron mobility transistors. The Pennsylvania State University

20. Wang ZL (2009) Energy harvesting using piezoelectric nanowires - a correspondence on Enegry Harvesting Using Nanowires? by Alexe et al. Adv Mater 21: 1311

21. Wang ZL (2008) Towards self-powered nanosystems: from nanogenerators to nanopiezotronics. Adv Mater 18: 3553

22. Fang $\mathrm{TH}$, Kang SH (2010) Physical properties of $\mathrm{ZnO}: \mathrm{Al}$ nanorods for Piezoelectric nanogenerator application. Curr Nanosci 6: 505

23. Kim DY, Son JY (2009) Horizontal ZnO nanowires for gas sensor application: Al-doping effect on sensitivity. Electrochem Solid State Lett 12: 1109

24. Chen CQ, Shi Y, Zhang YS, Zhu J, Yan YJ (2006) Size dependence of young's modulus in ZnO nanowires. Phys Rev Lett 96: 075505

25. Dai L, Cheong WC, Sow CH, Lim CT, Tan VB (2010) Molecular dynamics simulation of ZnO nanowires: size effects, defects, and super ductility. Langmuir 26: 1165

26. Tong H, Wang B, Ou-Yang Z (2008) Electric potential generated in ZnO nanowire due to piezoelectric effect. Thin Sold Films 516: 2708

doi:10.1186/2213-9621-1-4

Cite this article as: van den Heever and Perold: The influence of resistance and carrier concentration on the output voltage of a $\mathrm{ZnO}$ nanogenerator. Micro and Nano Systems Letters 2013 1:4.

\section{Submit your manuscript to a SpringerOpen ${ }^{\circ}$ journal and benefit from:}

- Convenient online submission

- Rigorous peer review

- Immediate publication on acceptance

- Open access: articles freely available online

- High visibility within the field

- Retaining the copyright to your article

Submit your next manuscript at $\boldsymbol{~ s p r i n g e r o p e n . c o m ~}$ 